\title{
Psychopharmacology and Intellectual Disability: The Continuing Dilemmas of "Challenging Behaviour"
}

\section{Highlights}

- There appears to be little advantage in continuing to distinguish "challenging behaviour" from problem behaviours as an identifiable category of mental disorder.

- The biopsychosocial perspective is a key consideration in formulating care plans for these individuals.

- To counter a longstanding pattern of overuse of antipsychotic drugs, contemporary practice guidelines foster a conservative approach to prescribing psychotropic medications.

- A regional tertiary care program can address the complex needs of these individuals, as well as related training and manpower development responsibilities for caregivers and care providers.

Keywords: Intellectual disability, Biopsychosocial, Antipsychotics, Dual diagnosis, Mental illness.

Received: September 17, 2015, Accepted: December 23, 2015, Published: December 28, 2015

\section{Introduction}

The use of psychotropic medications in the management of problem behaviours in persons with an intellectual disability tends to generate professional controversy and public misunderstanding. Medications are often perceived as "chemical restraints" and their prescribers as neglectful of expected professional standards and obligations. This paper, based on a review of relevant published information, endeavors to provide a balanced perspective that will ensure optimal care, including the prescription of psychotropic medications as needed, by health professionals. Diagnosis and categorization of mental disorders in persons with intellectual disabilities, the biopsychosocial perspective in formulating care plans, inter professional collaboration and manpower problems, and risk-benefit issues in the selection and monitoring of various medications are among the topics to be considered.

\section{Highlights}

\section{Diagnosis and categorization of mental disorders}

The term "challenging behaviour" was introduced in the mid 1980 's by UK psychologist [1] during community resettlement of individuals who had been institutionalized. Referring to those
D McCreary ${ }^{1}$ and Muhammad Ayub²

\section{Professor Emeritus, Division of Developmental Disabilities, Department of Psychiatry, Queen's University, Kingston, Canada \\ 2 Division of Developmental Disabilities, Associate Professor, Department of Psychiatry, Queen's University, 191 Portsmouth Avenue Kingston, K7M8A6 Kingston, Canada}

Corresponding author:

Muhammad Ayub

Division of Developmental Disabilities, Associate Professor, Department of Psychiatry, Queen's University, 191 Portsmouth Avenue Kingston, K7M8A6 Kingston, Canada.

\section{Đma84@queensu.ca}

Tel: $1+(613)$ 549-7944 with a range of problem behaviours, particularly aggression and self injury, the "challenge" was accommodating these behaviours while providing a "normal" life in local communities (now often called social inclusion). Clinical investigations of persons with intellectual disabilities over the next several years $[2,3]$ revealed that a number of mental disorders, medical problems and environmental factors contributed to "challenging behaviour". This complexity is reflected in [4] review of functional behavioural assessments. In conducting these assessments Tassé recognized the need for a comprehensive multi-method approach involving personal/social history, medical history and physical examination, evaluation of current medication including possible side effects or interaction effects, and psychiatric evaluation. In spite of the broadened scope for functional behavioural assessments, the main focus remains behavioural and the goal for intervention some form of behaviour management.

Meanwhile, also in the UK, the Royal College of Psychiatrists published DC-LD, an innovative approach to diagnosis and categorization of mental disorders in persons with learning disabilities/mental retardation [5]. DC-LD accommodated the pathoplastic effects of cognitive impairment on the clinical features of mental disorders and the inclination of these individuals to communicate subjective distress of whatever origin behaviourally. 
It clustered mental disorders in five levels with level four devoted to problem behaviours, primary problem behaviours or those secondary to medical problems or to other mental disorders. Accordingly, DC-LD identified "challenging behaviours" as arising from several possible origins without implying that management was necessarily "behavioural". Indeed, management, depending on the results of the assessment, might include a number of options, sometimes behavioural, sometimes psychotropic medications and sometimes both simultaneously. As noted more recently by [6] both behavioural and pharmacological treatments have been shown to work in symptom reduction, but there has been limited investigation of combined treatment, particularly investigations of constructs such as impairment, adaptive behaviour, and quality of life.

\section{The biopsychosocial perspective in formulating care plans}

Sometimes the origin of a challenging behaviour is immediately obvious to a health or mental health professional with some understanding of the biopsychosocial perspective. Examples might include a dental abscess in an individual recently observed by a caregiver to be striking his jaw, an irritable and sleepless group home resident whose mother died recently, or a young adult school-leaver frustrated because there is no job or training centre available. In these instances the importance of biological, psychological or social factors in planning remedies is reasonably clear. Unfortunately in many situations the origin of challenging behaviour is less obvious, although the need to consider the possibilities from a biopsychosocial perspective remains[7]. Many individuals with intellectual disabilities have multiple health problems (e.g., congenital anomalies, sensory problems, seizure disorders). Many are exposed to recurring psychological stressors (e.g., teasing, bullying, abuse) doubly traumatizing since they may not understand how to report these transgressions and engage others for help. And many are exposed to social neglect (e.g., training/employment opportunities, recreational activities). Frequently, biological, psychological and social stressors may operate synergistically, implying that a care plan to be successful will require far more than prescription of a psychotropic medication. To conclude otherwise would suggest that the professional consulted for help is either poorly trained [8] or is practicing in circumstances where the provision of acceptable care is impossible for other reasons, or both.

\section{Inter professional collaboration and manpower problems}

As knowledge about problem behaviours in those with intellectual disabilities and how to manage them gradually expands, it is becoming clear that interprofessional collaboration and the availability of a skilled workforce are key considerations.

To illustrate these issues this section outlines three practice scenarios described in the contemporary professional literature, maintaining a focus on the role of psychopharmacology as addressed in relevant clinical practice guidelines.

The Canadian Consensus Guidelines concerned with the Primary
Care of Adults with Developmental Disabilities make the following assertions [9]:

- Problem behaviour, such as aggression and self injury is not a psychiatric disorder but might be a symptom of a healthrelated disorder or other circumstances (e.g.,insufficient supports)

- Despite the absence of an evidence base, psychotropic medications are regularly used to manage problem behaviours among adults with ID. Antipsychotic drugs should no longer be regarded as an acceptable routine treatment of problem behaviours in adults with ID.

\section{As well, the following recommendations are provided:}

- Investigate relevant causes for problem behaviour (physical, environmental, emotional) before considering a psychiatric diagnosis.

- Facilitate "enabling environments" for the individual.

- Plan for a functional analysis and interdisciplinary understanding of problem behaviours.

- Regularly audit the use of prescribed psychotropic medications, including those" as needed".

- Consider reducing and stopping, at least on a trial basis medications not prescribed for a specific psychiatric diagnosis.

The World Psychiatric Association has published a guide concerned with psychotropic drugs and problem behaviours to provide "clinicians and carers of adults with ID worldwide with good practice advice despite the lack of good quality evidence"[10] of note is the conclusion "it is not possible to recommend the type of medication for the treatment of specific problem behaviours because there is no evidence to support such specificity".

Accordingly, much of the content is related to procedural issues:

- An attempt should be made to secure multidisciplinary input throughout the process of assessment and management.

- Non-medication management should be considered first and sometimes medication may be needed either on its own or as an adjunct to non-medication based management.

- The effectiveness and possible adverse effects of the prescribed medication should be monitored at regular intervals.

- Non-medication management strategies and the withdrawal of medication should be considered at regular intervals.

- Try to stabilize the person's problem behaviour on a minimum number of medications prescribed at the lowest possible dose.

Both guidelines imply the need for skilled manpower and for interprofessional collaboration and they emphasize nondrug interventions, and if drugs are to be used, avoidance of polypharmacy and high doses.

A 2004 investigation of psychotropic drug use for persons with intellectual disabilities in the Norwegian county of Hedmark provides a useful backdrop for considering interprofessional collaboration and manpower problems [11]. One hundred and 10 (37.4\%) of 300 individuals surveyed were receiving a psychotropic 
drug; most received an antipsychotic over several years. Almost two thirds of the prescriptions were completed by general practitioners and a behavioural/environmental intervention had predated medication in only $7.4 \%$ of cases. Prescriptions provided by consulting psychiatrists working with a multidisciplinary team followed a behavioural/environmental intervention in $49.1 \%$ of cases; the authors concluded consulting psychiatric and behavioural expertise should be strongly recommended, or even required "in spite of the scarcity of such resources".

A report focused on evaluation of positive behavioural support [12] from Roscommon, Ireland provides another perspective. In this study outcomes were evaluated following behavioural interventions designed and implemented by frontline caregivers trained by clinical psychologists. Behaviours reduced to near zero levels following positive behavioural support and the use of psychotropic medications was reduced by $66 \%$. This study, although involving only 5 subjects, clearly demonstrates the value of interprofessional collaboration and of targeted staff training, in this instance of frontline caregivers.

The third report selected for consideration illustrates optimal arrangements for interprofessional and interagency collaboration, while simultaneously providing relevant training. The MHiLD program $[13,14]$ has evolved in southeast London over the past 30 years. It supports the efforts of caregivers and care providers in a region of almost 500,000 through "subspecialty" ambulatory clinics, inpatient treatment and training about "dual diagnosis" (including problem behaviours and the use of psychotropic drugs in care plans). The MHiLD, therefore, provides tertiary care in a fashion commonplace in contemporary society for those with cancer or heart disease. Those who might be concerned about the cost of tertiary care for person with a dual diagnosis are reminded of the results of a "cost of illness" study in the Netherlands [15] showing that intellectual disability leads all other health or mental health disorders, including cancer and heart disease.

\section{Risk-benefit considerations in prescribing psychotropic drugs}

The reader familiar with clinical practice involving individuals with intellectual disabilities and problem behaviours, in spite of the cautions outlined in previous sections, will appreciate that psychotropic drugs have a role, sometimes an important role, in managing selected cases referred for care. The Canadian Consensus Guidelines have transposed an "interpretation" from a widely quoted double blind placebo-controlled study of antipsychotic medications: "Antipsychotic drugs should no longer be regarded as a routine treatment of problem behaviours in adults with ID. There are many antipsychotic preparations besides those utilized in the study (i.e., halperidol, risperidone) that are used in treating aggressive behaviour in other clinical populations and there is no particular reason why they should not be considered as treatment options for persons with ID. The balance of this section reviews three publications about drugs useful in managing problem behaviours in persons with an intellectual disability to illustrate risk-benefit considerations as related to the selection and use of such preparations.

A double-blind placebo controlled study of the use of baclofen in treating self-injurious behaviour in persons with severe intellectual disability appears to have been somewhat ignored in contemporary reviews. Baclofen, a gamma-aminobutyric acid analogue, in doses between $30-300$ mgms daily was shown to have dramatic effects in reducing or eliminating self-biting, scratching and banging, and sometimes, coexistent aggressive behaviour. Caregivers also described subjects were happier, more communicative, more cooperative, mood changes that occurred early in treatment. Transient drowsiness and anorexia were observed; for 2 of 16 patients' with epilepsy seizure control deteriorated requiring withdrawal of baclofen in one case. Baclofen is widely prescribed in general medicine as a muscle relaxant [16-18].

Clozapine, known as an "atypical" antipsychotic drug, is widely used to manage "treatment-resistant" psychoses in the general population, but has now been shown to benefit individuals with intellectual disabilities and self-injurious behaviour, aggression, property destruction and stereotypical behaviour. Unfortunately, approximately $1.5 \%$ of those treated develop agranulocytosis, potentially fatal and considered a medical emergency; additional potential side effects are seizures, weight gain, sedation and hyper salivation. Accordingly prescribers must be fully aware of side effects and their management, especially regular monitoring of white blood cell counts to ensure early recognition of agranulocytosis. The Guideline prepared by Sabaawi, Singh and deLeon in 2006 is obviously important in managing those treated with clozapine [19].

Finally, readers will be interested in a "systematic review" of the effectiveness of mood stabilizers and antiepileptic medication for the management of behaviour problems in adults with intellectual disability published by Deb and colleagues [20]. Lithium carbonate is observed to be particularly useful in managing aggressive behaviour and no particular problem with side effects emerged given the availability of established approaches for measuring serum levels. The evidence for anticonvulsant mood stabilizers (e.g., carbamazepine, valproate and topiramate) was less convincing; most of the studies are retrospective and included small number of participants.

\section{Summary and Conclusions}

This review has surveyed published sources to characterize continuing dilemmas related to "challenging behaviour" in persons with intellectual disabilities. As more has been learned since "challenging behaviour" was first described in the mid 1980's, the desirability of addressing the needs of these individuals from a biopsychosocial perspective has been recognized. Problem behaviours are now integrated in more traditional mental disorder nosologies and a longstanding pattern of overuse of antipsychotic medications has given way to care planning that incorporates a range of medical, psychological and environmental interventions. While some jurisdictions have recognized the need for tertiary care services (including their contributions to training and manpower development) in managing these "high needs" individuals, in others, philosophical attachments to mainstreaming in achieving social inclusion, have blocked these developments. 


\section{References}

1 Emerson E, Toogood A, Mansell J, et al. (1987) CHALLENGING BEHAVIOUR AND COMMUNITY SERVICES: Introduction and overview. $\mathrm{J}$ Br Inst Ment Handicap APEX 15: 166-169.

2 Bouras N, Drummond C (1992) Behavior and psychiatric disorders of people with mental handicaps living in the community. J Intellect Disabil Res JIDR 36: 349-357.

3 Moss S, Emerson E, Kiernan C, et al. (2000) Psychiatric symptoms in adults with learning disability and challenging behaviour. $\mathrm{Br} J$ Psychiatry177: 452-456.

4 Tassé MJ (2006) Functional behavioural assessment in people with intellectual disabilities. Curr Opin Psychiatry 19: 475-480.

5 Cooper S-A, Melville CA, Einfeld SL (2003) Psychiatric diagnosis, intellectual disabilities and Diagnostic Criteria for Psychiatric Disorders for Use with Adults with Learning Disabilities/Mental Retardation (DC-LD). J Intellect Disabil Res JIDR 47 Suppl 1: 3-15.

6 Lecavalier L (2012) Treating psychopathology in adults with developmental disabilities: glass half empty or half full? Can J Psychiatry Rev Can Psychiatr 57: 585-586.

7 McCreary B, Jones J (2015) Intellectual Disabilities and Dual Diagnosis, McGill-Queen's University

8 Werner S, Stawski M (2012) Mental health: Knowledge, attitudes and training of professionals on dual diagnosis of intellectual disability and psychiatric disorder. J Intellect Disabil Res 56: 291-304.

9 Sullivan WF, Berg JM, Bradley E, et al. (2011) Primary care of adults with developmental disabilities: Canadian consensus guidelines. Can Fam Physician Médecin Fam Can 57: 541-553, e154-168.

10 Deb S, Kwok H, Bertelli M, et al. (2009) International guide to prescribing psychotropic medication for the management of problem behaviours in adults with intellectual disabilities. World Psychiatry Off J World Psychiatr Assoc WPA 8: 181-186.
11 Holden B, Gitlesen JP (2004) Psychotropic medication in adults with mental retardation: prevalence, and prescription practices. Res Dev Disabil 25: 509-521.

12 McClean B, Grey IM, McCracken M (2007) An evaluation of positive behavioural support for people with very severe challenging behaviours in community-based settings. J Intellect Disabil JOID 11: 281-301.

13 Chaplin E, O'Hara J, Holt G, et al. (2009) Mental health services for people with intellectual disability: challenges to care delivery. $\mathrm{Br} \mathrm{J}$ Learn Disabil 37: 157-164.

14 Bouras N, Holt G (2004) Mental health services for adults with learning disabilities. Br J Psychiatry 184: 291-292.

15 Polder JJ, Meerding WJ, Bonneux L, et al. (2002) Healthcare costs of intellectual disability in the Netherlands: a cost-of-illness perspective. J Intellect Disabil Res JIDR 46: 168-178.

16 Tyrer P, Oliver-Africano PC, Ahmed Z, et al. (2008) Risperidone, haloperidol, and placebo in the treatment of aggressive challenging behaviour in patients with intellectual disability: a randomised controlled trial. Lancet 371: 57-63.

17 Stahl SM, Morrissette DA (2014) Stahl's Illustrated Violence: Neural Circuits, Genetics and Treatment. Abridged edition. Cambridge; New York: Cambridge University.

18 Primrose DA (1979) Treatment of self-injurious behavior with a GABA (gamma-aminobutyric acid) analogue. J Ment Defic Res23: 163-173.

19 Sabaawi M, Singh NN, Leon J de. Guidelines for the use of clozapine in individuals with developmental disabilities.

20 Deb S, Chaplin R, Sohanpal S, et al. (2008) The effectiveness of mood stabilizers and antiepileptic medication for the management of behaviour problems in adults with intellectual disability: a systematic review. J Intellect Disabil Res JIDR 52: 107-113. 